Artículos científicos

\title{
Impacto de la asignatura Servicio Social dentro del programa educativo de la licenciatura en Enfermería
}

Impact of the Social Service Subject Within the Educational Program of the Nursing Degree

Impacto da disciplina Serviço Social no programa educacional do curso de Enfermagem

\author{
Alma Delia Lara Cerda \\ Universidad Autónoma de San Luis Potosí, México \\ almalara73@hotmail.com \\ https://orcid.org/0000-0001-5293-8058 \\ Nereyda Hernández Nava \\ Universidad Autónoma de San Luis Potosí, México \\ nereyda.hernandez@uaslp.mx \\ https://orcid.org/0000-0002-4965-1045 \\ Alejandro Martínez Ramírez \\ Universidad Autónoma de San Luis Potosí, México \\ alejandro.martinez@uaslp.mx \\ https://orcid.org/0000-0001-8292-0377 \\ Rosa Eréndira Fosado Quiroz \\ Universidad Autónoma de San Luis Potosí, México \\ rosa.fosado@uaslp.mx \\ https://orcid.org/0000-0002-5344-7274 \\ Ma del Rocío Rocha Rodríguez \\ Universidad Autónoma de San Luis Potosí, México \\ rocio@uaslp.mx \\ https://orcid.org/0000-0002-3714-3967
}




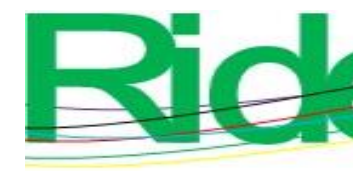

Revista Iberoamericana para la Investigación y el Desarrollo Educativo ISSN 2007 - 7467

\section{Resumen}

En México, el servicio social en las universidades es una actividad temporal y obligatoria para los estudiantes. El programa educativo de la licenciatura en Enfermería de la Coordinación Académica Región Altiplano de la Universidad Autónoma de San Luis Potosí (UASLP) incorporó la asignatura de Servicio Social como un espacio de formación y de acompañamiento para los alumnos prestadores de servicio social. El objetivo del presente trabajo fue evidenciar el impacto de la asignatura en cuestión en la formación de los alumnos de la licenciatura en Enfermería. Se trata de una investigación descriptiva y transversal. Se analizaron cuatro generaciones, del 2015 al 2018, con 166 alumnos, y se contrastaron las acciones educativas del programa con los resultados del Examen General de Egreso de Enfermería aplicado por el Centro Nacional para la Evaluación de la Educación Superior (Ceneval). En cada una de las generaciones, se incorporaron diferentes estrategias educativas a partir de exámenes diagnósticos a los alumnos de la materia Servicio Social. De las cuatro generaciones en estudio, 2015 a 2018, se obtuvieron resultados con testimonio de satisfactorio y con testimonio de sobresaliente: $93.3 \%, 73.3 \%, 75.0 \%$ y $84.3 \%$, respectivamente, cifras por encima de la media nacional del 2017 , que fue de $53.2 \%$. Al evidenciar, a través de una prueba estandarizada, los alcances significativos en la evaluación de los cuatro desempeños que son considerados por el Ceneval, a saber, "Educación y promoción de la salud", "Cuidados integrales al paciente", "Técnica y procedimientos y “Gestión, administración e investigación”, se ha demostrado el impacto positivo que la asignatura de Servicio Social tiene en el programa de la licenciatura en Enfermería de la UASLP.

Palabras clave: educación en enfermería, examen general de egreso, recursos humanos en salud, servicio social.

\section{Abstract}

In Mexico, social service in universities is a temporary and compulsory activity for students. The educational program of the degree in Nursing of the Coordinación Académica Región Altiplano of the Universidad Autónoma de San Luis Potosí (UASLP) incorporated the Social Service subject as a training and support space for students providing social service. The objective of this work was to show the impact of the subject in question on the training of 


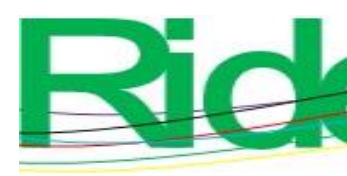

Revista Iberoamericana para la Investigación y el Desarrollo Educativo

ISSN 2007 - 7467

students of the Nursing degree. It is a descriptive and transversal investigation. Four generations were analyzed, from 2015 to 2018, with 166 students, and the educational actions of the program were contrasted with the results of the General Nursing Exit Exam applied by the Centro Nacional para la Evaluación de la Educación Superior (Ceneval). In each of the generations, different educational strategies were incorporated from diagnostic tests to the students of the Social Service subject. Of the four generations under study, 2015 to 2018, results were obtained with a satisfactory testimony and with an outstanding testimony: $93.3 \%, 73.3 \%, 75.0 \%$ and $84.3 \%$, respectively, figures above the national average of 2017 , which was $53.2 \%$. By demonstrating, through a standardized test, the significant achievements in the evaluation of the four performances that are considered by Ceneval, namely, "Education and health promotion", "Comprehensive patient care", "Technique and procedures and "Management, administration and research", the positive impact that the Social Service subject has on the UASLP Nursing degree program has been demonstrated.

Keywords: education in nursing, general exam for graduates, human resources in health, health services, social service.

\section{Resumo}

No México, as instituições de ensino que formam graduados em enfermagem têm o serviço social como atividade temporária e obrigatória. O programa pedagógico da Licenciatura em Enfermagem da Coordenação Académica da Região Altiplano da Universidade Autónoma de San Luis Potosí incorporou a disciplina de Serviço Social como um espaço de formação no qual é prestado apoio aos alunos que prestam serviço social durante a sua realização. O objetivo deste trabalho foi demonstrar a abrangência da disciplina Serviço Social na formação dos alunos provedores da mesma na Licenciatura em Enfermagem, utilizando como ferramenta indireta a avaliação nacional dirigida aos egressos desta profissão. Esta pesquisa foi realizada de forma descritiva e transversal, na qual foram revisados os resultados da implantação do Serviço Social como disciplina. Foram analisadas quatro gerações, de 2015 a 2018, com 166 alunos, onde as ações educativas do programa foram comparadas com os resultados do Exame Geral de Saída de Enfermagem aplicado pelo Centro Nacional de Avaliação do Ensino Superior. Diferentes estratégias educacionais foram incorporadas a partir de testes diagnósticos aos alunos do Serviço Social, em cada uma das gerações, com 


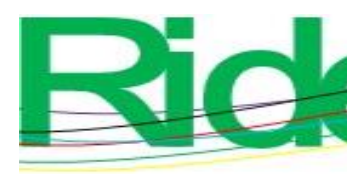

Revista Iberoamericana para la Investigación y el Desarrollo Educativo

ISSN $2007-7467$

impacto positivo nos resultados da avaliação externa nacional. Das quatro gerações em estudo, 2015 a 2018, os resultados foram obtidos com testemunho satisfatório e com depoimentos marcantes: $93.3 \%, 73.3 \%, 75,0 \%$ e $84.3 \%$; respectivamente, estando todos acima da média nacional de 2017, que foi de 53.2\%. Com esses resultados, ficou demonstrado o impacto que a disciplina de Serviço Social tem no programa educacional do Curso de Graduação em Enfermagem, ao mostrar por meio de um teste padronizado os avanços significativos na avaliação dos quatro desempenhos considerados por este teste: educação e promoção da saúde, atendimento integral ao paciente, técnica e procedimentos e gerenciamento, administração e pesquisa.

Palavra-chave: educação em enfermagem, exame geral para graduados, recursos humanos em saúde, serviços de saúde, serviço social.

Fecha Recepción: Septiembre $2020 \quad$ Fecha Aceptación: Febrero 2021

\section{Introducción}

El servicio social es aquella acción profesional dispuesta a contribuir en el desarrollo de un grupo, comunidad o institución, en la medida que se realiza alguna actividad o intervención en favor de las personas (Cano, 2004). En México, el servicio social surgió para dar cobijo, con los conocimientos y servicios de los futuros profesionales que se estaban formando en las universidades, a las clases más desprotegidas del país, y adquiere la característica de obligatorio durante el gobierno del presidente general Lázaro Cárdenas en su gestión, 1934 a 1940 (Ramírez, 2012), y no solo se presta en beneficio de la sociedad, sino también del Estado. Es por esto por lo que se reviste de una connotación moral y ética relacionada con la solidaridad social. Además, para muchas instituciones educativas, es el único medio de retribuir a la sociedad el apoyo económico recibido para su creación y desarrollo, así como para los alumnos el medio para compensar el privilegio de haber recibido educación gratuita escolarizada.

Las leyes que lo rigen se han reformado, pero sigue siendo necesario realizarlo a fin de obtener el título académico o el grado profesional como técnico, técnico superior o profesional con licenciatura (Presidencia de la República, 30 de marzo de 1981). En nuestro país sigue siendo uno de los medios, junto a las prácticas profesionales, que se realizan también tanto en instituciones públicas como privadas, para complementar la formación o la educación integral y apoyar las acciones y actividades que deben cumplir maestros y alumnos 


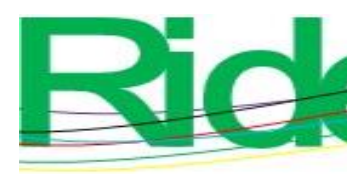

Revista Iberoamericana para la Investigación y el Desarrollo Educativo ISSN $2007-7467$

a fin de lograr los desempeños que los llevarán a alcanzar las competencias propuestas en los planes de estudio (Cano, 2004).

Por su creciente aportación en los procesos de mantenimiento o recuperación de la salud del individuo, de la familia o de la comunidad en las diferentes etapas de la vida, la enfermería ha resultado ser una disciplina fundamental en el equipo de salud (Alvarado, 2012; Balderas, 2012). La formación académica del personal de enfermería evoluciona a la par del avance tecnológico de los servicios de salud, del país y al orden internacional (Marriner, 1993, 1997). Esto con el objetivo de ofrecer servicios de salud de calidad y acordes a los diferentes roles que ejerce el profesional de enfermería en las distintas áreas: administración hospitalaria, capacitación, atención preventiva, atención especializada, entre otras, así como en la labor investigativa para la solución de los problemas de salud que afectan a la población (Marriner, 2009).

El cuidado de enfermería consiste en hacer por alguien lo que no puede hacer la persona por sí misma con respecto a sus necesidades básicas, incluye también la capacitación para mejorar la salud a través de la enseñanza, la comprensión de la expresión de sentimientos, la contribución para mejorar la calidad de la vida del enfermo y su familia ante la nueva experiencia que debe afrontar (Secretaría de Salud, 2 de septiembre de 2013).

El servicio social en enfermería es el período académico de proyección social que se desarrolla en unidades de primero, segundo y tercer nivel de atención y en el que el estudiante aplica los conocimientos, destrezas y aptitudes adquiridas en los ciclos escolarizados (Universidad Autónoma de San Luis Potosí [UASLP], 2013). De igual manera, favorece a que el estudiante adquiera las habilidades y las destrezas necesarias para profesionalizarse y la seguridad para cada una de sus intervenciones en el ámbito asistencial, docente, administrativo y de investigación. Para apoyar la implementación del servicio social se establecieron estrategias en el Programa operativo anual de servicio social de la licenciatura en Enfermería de la Coordinación Académica Región Altiplano [Coara] (2017, 2018), y la normativa que lo sustenta proporciona al prestador del servicio social la regulación y un punto de referencia y organización para alcanzar las metas previstas (UASLP, 2013).

El plan de estudios de la licenciatura en Enfermería de la Coara tiene una asignatura denominada Servicio Social, la cual cuenta con un programa-guía para que el docente apoye al alumno en el proceso de inserción y durante desarrollo de su servicio social en las instituciones de salud y asistencia social, empresas y centros educativos. Esta materia 


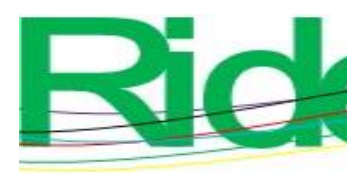

Revista Iberoamericana para la Investigación y el Desarrollo Educativo

ISSN 2007 - 7467

también tiene como objetivo fortalecer los conocimientos adquiridos para el logro del perfil de egreso, el cual se puede evidenciar a través de los resultados alcanzados por los egresados en la prueba nacional estandarizada de conocimientos conocida como Examen General para el Egreso de la Licenciatura en Enfermería (EGEL-Enfer), aplicada por el Centro Nacional de Evaluación para la Educación Superior (Ceneval). Cabe señalar que, en primer lugar, el Ceneval (2018a) es un organismo evaluador externo; y en segundo, que el EGEL-Enfer permite identificar el nivel de dominio o desempeño logrado por el sustentante con respecto a los conocimientos y habilidades que el consejo técnico del examen ha definido como necesarios para iniciarse eficazmente en el ejercicio profesional.

El Informe Anual de Resultados 2017 del EGEL-Enfer indica que 53.2\% de los alumnos que presentaron el examen obtuvieron testimonio de desempeño satisfactorio o sobresaliente, $39.8 \%$ y $13.4 \%$, respectivamente, y $46.8 \%$ no obtuvieron el testimonio (Ceneval, 2017), todos estos alumnos estaban activos, ejercían o habían ejercido como egresados en esta área de la salud, con el respaldo de la normatividad correspondiente y formando parte del personal de las instituciones de salud (Ceneval, 2018b). Esta misma percepción la manifiestan los pacientes cuando son atendidos: al distinguir que son los prestadores de servicio social quienes a menudo los atienden, solicitan que los profesionales del cuidado estén capacitados (Mendoza, 2017).

Esta inquietud por alcanzar los niveles de correspondencia entre la formación profesional y el desempeño en el servicio social de los alumnos de la licenciatura en Enfermería es compartida con otras universidades del país (Arriaga, Cruz, Morales y Vicente, 2016; Carmona, González y Crespo, 2012; Sánchez, Guzmán y Baeza, 2018). En esa línea, Navarrete, Barrera y Martín (2010) proponen fortalecer la formación integral de los prestadores de servicio social, reforzar actitudes solidarias con las comunidades y mejorar la articulación de las funciones sustantivas de las instituciones de educación superior por medio del servicio social.

Por lo tanto, el objetivo de este trabajo es evidenciar el alcance de la materia Servicio Social en la formación de los alumnos prestadores de este en la licenciatura en Enfermería. La evaluación nacional dirigida a los egresados de la carrera servirá como herramienta indirecta para alcanzar dicho propósito. 


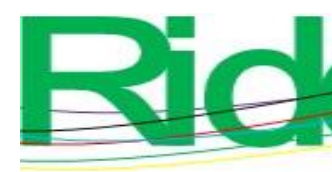

Revista Iberoamericana para la Investigación y el Desarrollo Educativo ISSN 2007 - 7467

\section{Método \\ Tipo o diseño del estudio}

La investigación es cuantitativa, descriptiva y transversal. Se revisaron y contrastaron los resultados de los exámenes en la asignatura de Servicio Social y del EGEL-Enfer realizado por las generaciones que egresaron en el 2015, 2016, 2017 y 2018, tal y como se ha realizado en otros estudios de otras instituciones (Barrera, Canepa y Santiago, 2016).

\section{Escenarios}

La asignatura de Servicio Social forma parte de la licenciatura en Enfermería, se ubica en el noveno y décimo semestre del plan de estudios vigente y tiene una duración de 12 meses con 60 créditos (UASLP, 2013). Así, cumple con el artículo 16 del Reglamento de Servicio Social de la UASLP, a saber:

La duración del servicio social no deberá ser menor de 480 horas, mismas que se cubrirán en un período que corresponda a seis meses continuos, ni podrá ser mayor de 960 horas cubiertas en el transcurso de doce meses continuos para el programa o proyecto, que por razón de su naturaleza tenga que operar continuamente, se apegará al reglamento interno o lo establecido en el plan de estudios respectivo (UASLP, 2015b, pp. 6).

Asimismo, al ser de carácter obligatorio, cumple con el artículo 2 del mismo reglamento, que lo posiciona como un requisito para la titulación de licenciatura y como una experiencia complementaria del aprendizaje (UASLP, 2015a).

La asignatura es teórica-práctica y transcurre en ambientes reales. Los alumnos asisten a las instituciones de salud de primero y segundo nivel de atención, así como a las empresas de nuestra comunidad o ciudades foráneas, también apoyan a instituciones de asistencia social y de educación. Por lo tanto, el aprendizaje se establece en una modalidad mixta, presencial y virtual. En la primera modalidad, el prestador del servicio social realiza la actividad en escenarios reales, en donde tiene contacto con los usuarios del sistema de salud o usuarios que requieren atención y cuidado a la salud, además de contacto con los diferentes profesionales del área de la salud que lo guían en esta etapa de su formación. En la modalidad virtual, el alumno recibe un aprendizaje contextualizado y significativo de acuerdo con las experiencias que está atendiendo en los escenarios reales, con estrategias de 

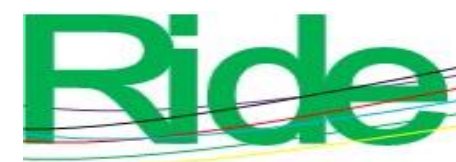

Revista Iberoamericana para la Investigación y el Desarrollo Educativo

ISSN 2007 - 7467

aprendizaje en proyectos, problemas, casos y solución de conflictos. En suma, se trata de un aprendizaje activo, transformador y colaborativo que lo forma y lo fortalece como futuro profesional de enfermería (UASLP, 2015a, 2017).

El EGEL-Enfer es una prueba nacional, especializada y estandarizada, que evalúa los conocimientos y habilidades que son críticos para ejercer como profesional de la enfermería. El requisito para presentarlo es cubrir $100 \%$ de los créditos de la licenciatura o, en su caso, estar cursando el último semestre de la carrera (Ceneval, 2018a). El examen consta de dos sesiones con duración de cuatro horas cada una; debe realizarse en un espacio con servicio de informática, cómodo y ventilado. Para la licenciatura de Enfermería de esta institución, el EGEL-Enfer es un requisito indispensable para obtener el grado y lo presentan cuando se concluye el servicio social.

\section{Población}

La asignatura de Servicio Social se ha impartido en cuatro ocasiones, un impacto directo sobre 166 alumnos inscritos a esta materia, quienes, al concluir la asignatura, han realizado el EGEL-Enfer (tabla 1).

Tabla 1. Historial de los alumnos que cursaron la asignatura de Servicio Social en el noveno y décimo semestre de la carrera de Enfermería

\begin{tabular}{|l|c|c|c|c|c|}
\hline Generación & $2010-2015$ & $2011-2016$ & $2012-2017$ & $2013-2018$ & Total \\
\hline Alumnos & 37 & 43 & 35 & 51 & 166 \\
\hline
\end{tabular}

Fuente: Elaboración propia

\section{Criterios de selección de la población de estudio}

El criterio de inclusión fue que todos los alumnos concluyeran el curso Servicio Social y, al concluirlo, presentaran el EGEL-Enfer. El criterio de exclusión fue aplicado a quienes no acreditaran la asignatura o la dieran de baja, o bien no presentaran el EGEL-Enfer. 


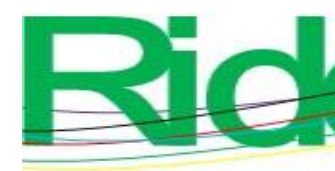

Revista Iberoamericana para la Investigación y el Desarrollo Educativo ISSN 2007 - 7467

\section{Definición de la población}

La población fue el total de cuatro generaciones de la licenciatura en Enfermería, que se inscribieron y concluyeron la asignatura de Servicio Social y presentaron el EGEL-Enfer, esto es, 166 alumnos.

\section{Colecta de datos}

Los resultados obtenidos en la asignatura de Servicio Social y en el EGEL-Enfer fueron colectados identificando al alumno y la generación a la cual pertenece; a través de la expresión numérica obtenida en las diferentes actividades y contrastada con el resultado obtenido del EGEL-Enfer.

La asignatura de Servicio Social tiene una única promoción: es anual e inicia el 1 de agosto y finaliza el 31 de julio del año siguiente. La primera generación lo cursó en el 2014, las posteriores generaciones atendieron en el año 2015, 2016 y 2017. La asignatura se organiza con una sesión mensual, presencial y obligatoria de ocho horas en las instalaciones de la escuela, y el objetivo de cada sesión es que el profesor a cargo asesore sobre los temas que orientan y preparan al alumno en los temas seleccionados en el programa académico (tabla 2) utilizando los problemas y casos reales que afrontan los alumnos en la cotidianidad de la prestación de su servicio social; además, en esta sesión se les aplica un examen diagnóstico de conocimientos que les permite autoevaluarse y reforzar su formación.

La materia se evalúa a través de cuatro exámenes parciales. El promedio de estos suscribe la acreditación o no acreditación de la asignatura. Las evaluaciones diagnósticas practicadas a los alumnos a partir de la tercera generación (2012-2017) ahora se revisten de mayor importancia, no solo son un marco para seleccionar los temas con mayor deficiencia y necesarios de fortalecer, sino que propician y guían la intervención educativa de un docente experto, quien brinda asesoría de forma asincrónica y sincrónica fuera de las 12 sesiones presenciales. 
Tabla 2. Programa académico de la asignatura de Servicio Social de la licenciatura en Enfermería de la Coara (UASLP, 2010)

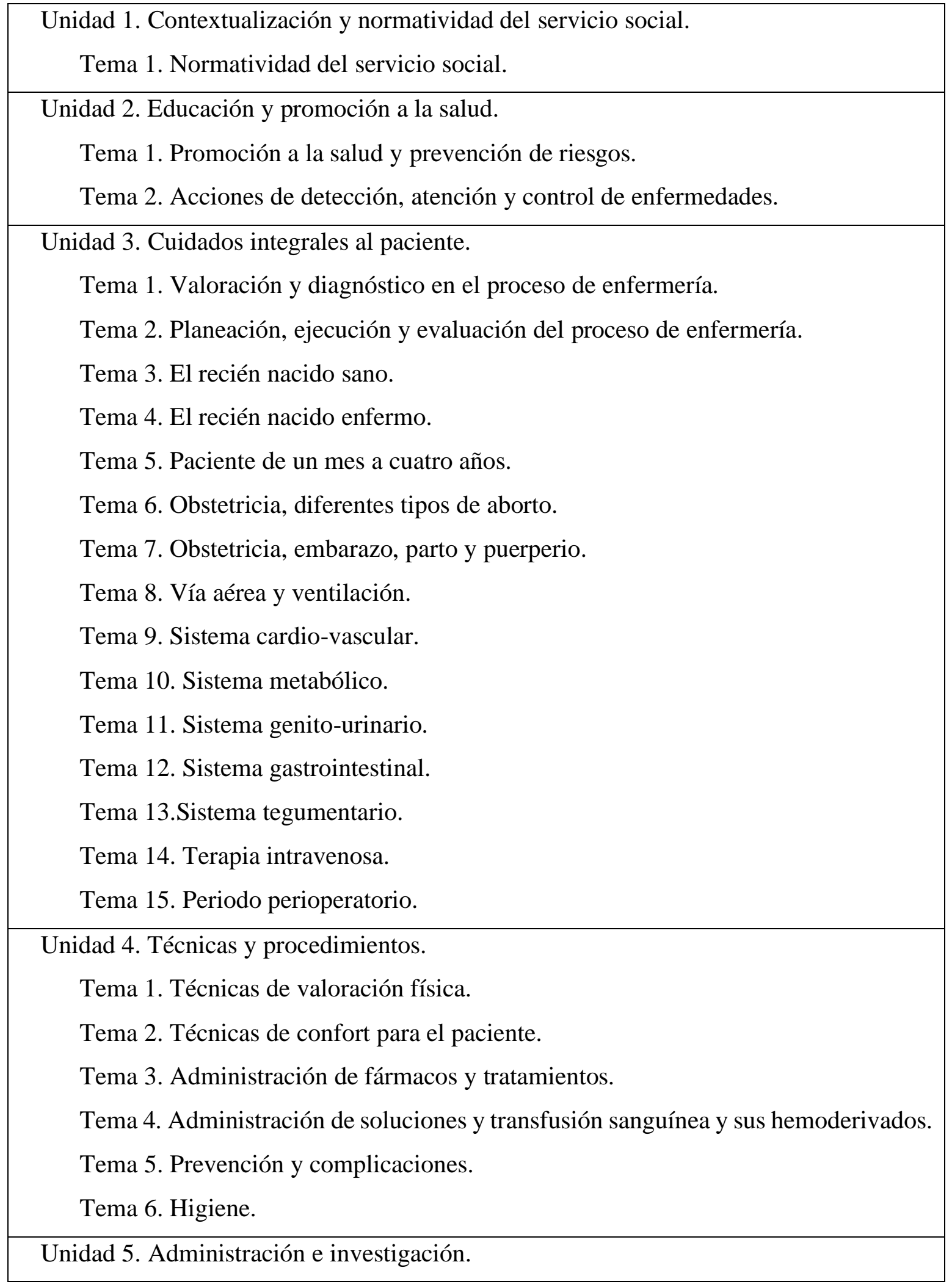




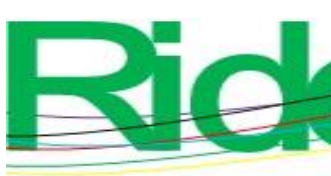

Revista Iberoamericana para la Investigación y el Desarrollo Educativo ISSN $2007-7467$

Tema 1. Gestión y administración de los recursos de enfermería.

Tema 2. Investigación.

\section{Fuente: Elaboración propia}

La evaluación diagnóstica para su diseño consideró la currícula de la licenciatura en Enfermería, la cual consta de 53 materias que se agrupan y fortalecen en cinco campos temáticos profesionalizantes y transversales: Enfermería, Biomédica, Humano social, Instrumental y Formación integral (UASLP, 2013, 2015a), así que para evaluar los conocimientos se realizó un examen diagnóstico con 166 reactivos que contemplan cada uno de estos campos. Pero el resultado final de la materia de Servicio Social considera solo dos tipos de evaluaciones, la autoevaluación y la heteroevaluación (con un asesor interno y un asesor externo). Los alumnos se evaluaron tres veces en el año y los asesores entregaron una calificación enriquecida con sus rúbricas personales.

La autoevaluación es una rúbrica que consideró tres apartados: la evaluación continua, el desempeño en el aprendizaje situado y la integración y procesamiento del trabajo colaborativo. Estas rúbricas reciben una ponderación equitativa, $33.3 \%$ cada una, y también incluyen el trabajo realizado por cada alumno a fin de integrar las competencias transversales del modelo educativo de la UASLP (2017): ético-valoral, comunicativa y de información, responsabilidad social y otras. Así, la primera consideró los reportes mensuales, examen y participación, además de la disciplina de los alumnos; la segunda, la asistencia y puntualidad, presentación, disciplina, colaboración, productividad, relaciones interpersonales e integración,y aplicación de conocimientos, y la tercera, la calidad, responsabilidad, colaboración, creatividad, disciplina, asistencia y puntualidad.

La evaluación interna consistió en una serie de exámenes periódicos que se aplicaron durante el servicio social, los cuales contemplaron los temas revisados en las 12 sesiones. Los resultados parciales debían permitir detectar las deficiencias de aprendizaje (conocimientos adquiridos) y apoyar la retroalimentación, la cual se realizó en el aula con la exposición de los temas y de forma virtual a través de grupos sociales privados, realizando lecturas y respondiendo dudas.

El trabajo académico se programó y revisó utilizando como herramienta el cronoplan anual, el cual se actualiza en cada generación, y contempla la entrega de un informe al término de cada sesión mensual, lo cual permite el seguimiento por parte de la academia de Servicio Social de la carrera. La actividad principal de aprendizaje de cada año ha consistido 


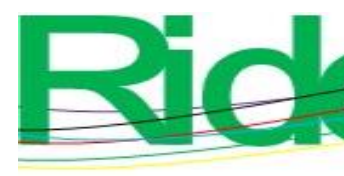

Revista Iberoamericana para la Investigación y el Desarrollo Educativo ISSN $2007-7467$

en exponer un caso clínico real con la metodología del proceso de cuidado enfermero. Y para fundamentar el caso los alumnos integraron tres diagnósticos de enfermería, los cuales debían cumplir con los requisitos de utilizar metas internacionales de seguridad del paciente, indicadores de calidad de enfermería, normas oficiales mexicanas, guías de la práctica clínica y técnicas de valoración especializada, así como dos procedimientos relacionados con el caso clínico, fundamentando la fisiopatología (Balderas, 2012; Marriner, 2009; Secretaría de Salud, 3 de enero de 2017; UASLP, 2017).

Para supervisar la formación cognitiva-instrumental de los alumnos se realizaron dos visitas a las unidades de adscripción, con autorización previa de la institución o empresa para acudir y entrevistar al asesor externo, a fin de evaluar la disciplina y los desempeños de los alumnos. Para la recolección de los datos, el programa de Servicio Social fue articulado por un profesor de asignatura, quien es responsable de esta área, y fue verificado por el coordinador de la carrera; el primero recolectó los datos de las evaluaciones, los procesó y promedió, para finalmente, con el segundo, analizar y concretar las nuevas estrategias. Y para medir el impacto del Servicio Social como asignatura los resultados del EGEL-Enfer se utilizaron como referencia (Pérez y Pinto, 2020).

El EGEL-Enfer se estructura con 162 reactivos, los cuales se distribuyen en cuatro áreas principales: "Educación y promoción de la salud", "Cuidados integrales al paciente”, “Técnicas, procedimientos” y "Gestión, administración e investigación”. A cada área se le otorga una puntuación y nivel de desempeño: 700-999 aún no satisfactorio, 1000-1149 satisfactorio y 1150-1300 sobresaliente (Ceneval, 2017). Los resultados del EGEL-Enfer de cada generación son entregados a las dependencias educativas en formato electrónico. La lista contiene el nombre del alumno, el puntaje obtenido en cada una de las cuatro áreas de conocimiento y el resultado de desempeño, datos que son analizados por la academia de Servicio Social.

\section{Análisis y tratamiento de los datos}

Los datos se recogieron en una hoja de cálculo Excel y las variables de estudio para el análisis de la muestra por generación fueron: calificación final de la asignatura, los resultados de la evaluación diagnóstica y de la evaluación final (prueba de 166 reactivos), realizada en este periodo de la asignatura y los resultados por área del EGEL-Enfer. 


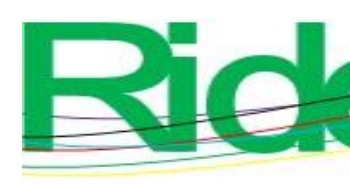

Revista Iberoamericana para la Investigación y el Desarrollo Educativo ISSN $2007-7467$

\section{Resultados}

Hasta el momento, esta materia se ha impartido a 166 alumnos pertenecientes a las cuatro generaciones; este estudio, sin embargo, se realizó a partir de la tercera generación. La primera herramienta que se aplicó a los alumnos fue el examen diagnóstico, con 166 reactivos propuestos en una ponderación de 0 a 10, para un valor 6.0 son necesarios 100 reactivos correctos. Los resultados obtenidos para la generación 2012-2017 es 6.3 ( \pm 0.05) y para la generación 2013-2018 es 6.2 ( \pm 0.05); $77.8 \%$ y $69.1 \%$ de los alumnos aprobaron de estas generaciones, respectivamente. Estos resultados invitaron a la autorreflexión: permitieron a los alumnos identificar sus áreas de oportunidad y contextualizar sus objetivos, por un lado, y permitieron a la academia redoblar esfuerzos para garantizar el perfil de egreso de estos y los futuros alumnos, por el otro. Se observó que los conocimientos teóricos estaban integrados a la práctica clínica, y los comentarios vertidos por sus asesores externos fueron: "Los alumnos en su mayoría trabajan de forma precisa y coherente con el contexto".

Las unidades de salud sedes de servicio social de segundo nivel de atención evaluaron con resultados satisfactorios a los alumnos. Lo anterior se confirmó en el periodo de preparación para la aplicación del examen final interno, ya que los alumnos contextualizaron correctamente los problemas caso, trabajaron de forma colaborativa en el aula y a través de medios virtuales, por medio de sesiones asincrónicas resolvieron conflictos, cada trabajo cumplió ampliamente con los requisitos que marca el programa; el objetivo de preparación se alcanzó cuando los alumnos, a través de lluvias de ideas, se retroalimentaron, repasaron los conocimientos teóricos adquiridos en semestres pasados e integraron estos en un proceso de solución.

El examen final interno obtuvo un mayor rendimiento. Los resultados obtenidos fueron: $8.6( \pm 0.05)$ para la generación 2012-2017 y $8.3( \pm 0.05)$ para la generación 20132018, en promedio debían lograr 133 reactivos correctos de 166 para un valor 8.0; $100 \%$ y $92.8 \%$ de alumnos aprobaron de estas generaciones, respectivamente (tabla 3). Estos resultados confirman el impacto positivo que tiene la asignatura de Servicio Social en el logro del perfil de egreso. Como se contrastó con los resultados de este examen, la generación 2012-2017 obtuvo 57.1 \% de testimonio en sus diferentes áreas, y la generación 2013-2018, $84.3 \%$. Las estrategias de mejora que se implementaron fueron: la impartición de un curso extraordinario con temas relevantes de enfermería, así como la organización y flexibilidad en la programación del curso a fin de garantizar la asistencia, e involucramiento de varios 


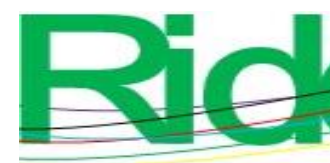

\section{Revista Iberoamericana para la
Investigación y el Desarrollo Educativo ISSN $2007-7467$}

maestros en la capacitación. Estas estrategias incrementaron los resultados de la evaluación externa EGEL-Enfer que se tomó como referencia para la valoración del impacto del curso Servicio Social.

\section{Discusión}

La asignatura de Servicio Social ha sido rediseñada de acuerdo con las necesidades detectadas en los alumnos. En el 2010 se creó esta asignatura y del 2015 en adelante se reajustó, específicamente la planeación para el desarrollo del programa. Los resultados nacionales del EGEL-Enfer 2017 muestran que, de los 16144 sustentantes, obtuvieron testimonio de desempeño sobresaliente $13.4 \%$, testimonio desempeño satisfactorio $39.8 \%$ y aún no satisfactorio $46.8 \%$; la suma de los porcentajes con testimonio sobresaliente y testimonio satisfactorio del reporte 2017 es de $53.2 \%$. En contraste, los alumnos sustentantes de las generaciones 2015 al 2018 de la licenciatura en Enfermería de la Coara han obtenido resultados en su mayoría por encima de la media nacional: $75.7 \%, 41.9 \%, 57.1 \%$ y $84.3 \%$, respectivamente (tabla 3). Es importante destacar que a partir de la tercera generación se incrementaron los testimonios sobresalientes, disminuyendo los resultados sin testimonio y los testimonios satisfactorios. Esto último nos indica que el nivel de desempeño se está homogeneizando favorablemente a través de las estrategias aplicadas en la asignatura.

Tabla 3. Resultados del examen de egreso de los alumnos de las cuatro generaciones de la licenciatura en Enfermería de la Coara

\begin{tabular}{|l|c|c|c|c|}
\hline \multirow{2}{*}{} & \multicolumn{4}{|c|}{$\begin{array}{r}\text { Generaciones de la licenciatura en Enfermería } \\
\text { (número de alumnos sustentantes y porcentaje del } \\
\text { resultado) }\end{array}$} \\
\hline Resultados del sustentante & $2010-2015$ & $2011-2016$ & $2012-2017$ & $2013-2018$ \\
\hline Aún no satisfactorio & $9(24.3 \%)$ & $25(58.1 \%)$ & $15(42.9 \%)$ & $8(15.7 \%)$ \\
\hline Desempeño satisfactorio & $22(59.5 \%)$ & $14(32.6 \%)$ & $15(42.9 \%)$ & $22(43.1 \%)$ \\
\hline Desempeño sobresaliente & $6(16.2 \%)$ & $4(9.3 \%)$ & $5(14.3 \%)$ & $21(41.2 \%)$ \\
\hline Excelencia & 1 & 0 & 1 & 4 \\
\hline Total de alumnos & 37 & 43 & 35 & 51 \\
\hline
\end{tabular}

Fuente: Elaboración propia 


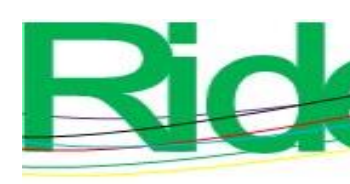

Revista Iberoamericana para la Investigación y el Desarrollo Educativo ISSN 2007 - 7467

En lo que respecta a testimonios del EGEL, el Ceneval instituyó un premio de excelencia académica a los sustentantes que alcanzan un nivel de desempeño excepcional en este examen. Los resultados del EGEL-Enfer en el 2017 muestran que 304 sustentantes obtuvieron dicha distinción, provenientes de 35 instituciones de educación superior, que representan $1.9 \%$. Es relevante mencionar que la carrera de Enfermería ha incrementado los resultados de alumnos con testimonio de excelencia académica de las generaciones en estudio: uno, cero, uno y cuatro alumnos, respectivamente, lo han obtenido; esto equivale a $2.7 \%, 0.0 \%, 2.9 \%$ y $7.8 \%$, lo cual muestra nuevamente estar por encima de la media nacional, por lo tanto, el impacto medido de manera indirecta a través de los resultados de EGEL-Enfer es positivo y verificable para la carrera de Enfermería.

\section{Conclusión}

El Servicio Social desde su creación y normalización siempre ha perseguido estos tres objetivos de forma general: 1) beneficiar a los sectores más desprotegidos de la sociedad apoyándose en el conocimiento científico y técnico existente en las instituciones de educación superior; 2) fortalecer la formación integral de los prestadores de servicio social para reforzar actitudes solidarias con las comunidades, y 3) fortalecer la articulación de las funciones sustantivas de las instituciones (Asociación Nacional de Universidades e Instituciones de Educación Superior [Anuies], 2012; Galván, 2009).

La asignatura de Servicio Social de la Coara no solo sumó estos objetivos en su creación, sino también consideró las normativas vigentes para la práctica de la enfermería en el Sistema Nacional de Salud y la educación en salud (Secretaría de Salud, 2 de septiembre de 2013, 3 de enero de 2017), en donde se establece los criterios para el ejercicio de la enfermería y la utilización de los establecimientos para la atención médica. Con esto se buscó apoyar la formación de recursos humanos que den respuesta de forma efectiva a las necesidades de la población en el área de salud. También, como siempre se ha buscado, que el programa de Servicio Social de la licenciatura en Enfermería de la Coara tuviera acciones colegiadas para que el estudiante cumpla las normativas, el perfil de egreso y apoye a consolidar la vinculación de la licenciatura con los sectores sociales.

El trabajo realizado impacta de manera objetiva, evidencia la formación que adquirieron los alumnos prestadores del servicio social, así como los conocimientos teóricosprácticos evaluados a través de un examen nacional. 


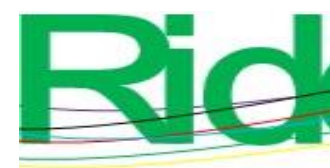

\section{Revista Iberoamericana para la Investigación y el Desarrollo Educativo ISSN 2007 - 7467}

La asignatura ha permitido integrar y fortalecer el aprendizaje del alumno, hacerlo interesante e interactivo a través de distintos medios, técnicas y estrategias de aprendizaje, colegiados en la academia de Servicio Social y con los alumnos; así, se ha promovido el desempeño con iniciativa propia y vocación por su profesión. Sin embargo, es deseable que la asignatura de Servicio Social se readapte al contexto epidemiológico y a las nuevas necesidades y urgencias de la comunidad a la cual se presta el servicio social. Por otro lado, es necesario que el programa logre disminuir el porcentaje generacional de alumnos sin testimonio a través de estrategias personalizadas de acuerdo con las necesidades de los alumnos.

La materia en cuestión permitió sistematizar el análisis de las experiencias que el alumno adquiere en los espacios reales; proponer estrategias de intervención, de atención, y de mejora en los diferentes espacios donde el alumno realiza el servicio social; dar seguimiento a los alumnos en los espacios donde están adscritos al servicio social y conocer las perspectivas del desempeño de los alumnos por parte de sus asesores externos; establecer áreas de mejora a el programa educativo de servicio social, e impactar favorablemente en los resultados de la evaluación externa EGEL-Enfer.

\section{Contribuciones a futuras líneas de investigación}

Las futuras líneas de investigación para continuar este proyecto serán: 1) realizar una evaluación cualitativa de la percepción de los clientes que atienden los alumnos durante el servicio social; 2) evaluar los resultados en el EGEL-Enfer obtenidos por estas generaciones y las siguientes y analizar el programa de la materia de Servicio Social para fortalecer el perfil de egreso; 3) proponer y evaluar nuevas herramientas de enseñanza-aprendizaje que fortalezcan el programa de la materia de Servicio Social; 4) realizar una evaluación cualitativa que recoja las experiencias de los alumnos y sus tutores o asesores en su estancia de servicio social; 5) rediseñar la materia de Servicio Social para que se adapte al contexto epidemiológico y a las nuevas necesidades y urgencias de la sociedad. Cada una de estas futuras líneas de investigación son aportes necesarios para fortalecer el servicio social y las competencias de los futuros profesionales de la enfermería. 


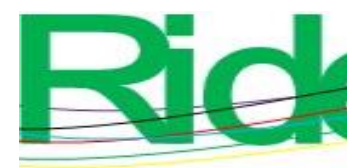

Revista Iberoamericana para la Investigación y el Desarrollo Educativo ISSN $2007-7467$

\section{Referencias}

Alvarado, A. (2012). Administración y mejora continua en enfermería. Ciudad de México, México: McGraw-Hill.

Arriaga, R., Cruz, A., Morales, F. y Vicente, P. Y. (2016). Desempeño de los prestadores de servicio social de licenciatura en enfermería de la DACS-UJAT en instituciones receptoras del sistema estatal de salud. Horizonte Sanitario, 15(1), 37-45. Recuperado de http://www.redalyc.org/pdf/4578/457845341005.pdf

Asociación Nacional de Universidades e Instituciones de Educación Superior [Anuies]. (2012). El servicio social de la educación superior. Punto de articulación con el entorno. Ciudad de México, México: Asociación Nacional de Universidades e Instituciones de Educación Superior.

Balderas, M. L. (2012). Administración de los servicios de enfermería (6. ${ }^{\mathrm{a}}$ ed.). Ciudad de México, México: McGraw-Hill.

Barrera, R. A., Canepa, A. A. y Santiago, J C. (2016). Desempeño de egresados de tecnologías de información en el examen general de egreso. Revista Iberoamericana para la Investigación y el Desarrollo Educativo, 7(13), 497-513. Recuperado de https://www.ride.org.mx/index.php/RIDE/article/view/261.

Cano, C. (2004). Reflexiones sobre el futuro del servicio social universitario. Reencuentro, (40), 1-10. Recuperado de http://www.redalyc.org/pdf/340/34004011.pdf.

Carmona, B., González, S. y Crespo, S. (2012). Opinión de los egresados del plan de estudios de la licenciatura en Enfermería de la Facultad de Estudios Superiores ZaragozaUniversidad Nacional Autónoma de México. Enfermería Global, (28), 180-191. Recuperado de http://revistas.um.es/eglobal/article/view/151971/138921.

Centro Nacional de Evaluación para la Educación Superior [Ceneval]. (2017). Informe Anual de Resultados 2017. Examen General para el Egreso de la Licenciatura en Enfermería. México: Dirección del Área de los Egel. Recuperado de https://www.ceneval.edu.mx/estadisticas-ceneval.

Centro Nacional de Evaluación para la Educación Superior [Ceneval]. (2018a). Examen General de Egreso de la Licenciatura en Enfermería. Recuperado de http://www.ceneval.edu.mx/enfermeria.

Centro Nacional de Evaluación para la Educación Superior [Ceneval]. (2018b). Guía para el sustentante. Examen General para el Egreso de la Licenciatura en Enfermería. 


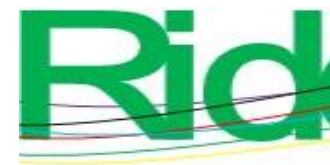

Revista lberoamericana para la Investigación y el Desarrollo Educativo ISSN 2007 - 7467

México: Dirección del Área de los EGEL. Recuperado de https://www.ceneval.edu.mx/documents/20182/34992/Guia_EGEL-

ENFER_26ed+26022018.pdf/91ff810f-bcb7-421c-9d33-c41fe2973b99.

Coordinación Académica Región Altiplano [Coara]. (2017). Programa operativo anual de servicio social de la licenciatura en Enfermería. San Luis Potosí, México: Coordinación Académica Región Altiplano.

Coordinación Académica Región Altiplano [Coara]. (2018). Programa operativo anual de servicio social de la licenciatura en Enfermería. San Luis Potosí, México: Coordinación Académica Región Altiplano.

Galván, J. L. (2009). Valoración global subjetiva. Recuperado de http://www.uacj.mx/ICB/redcib/Documents/Publicaciones/Valoración\%20Global\% 20Subjetiva.pdf.

Marriner, A. (1993). Manual para la administración de enfermería. Ciudad de México, México: McGraw-Hill.

Marriner, A. (1997). Administración y liderazgo de enfermería (5. ${ }^{a}$ ed.). Madrid, España: Elsevier.

Marriner, A. (2009). Guía de gestión y dirección en enfermería (8.a ed.). Barcelona, España: Elsevier Health Sciences.

Mendoza, G. Z. (2017). Programa de detección del cáncer cervicouterino: políticas públicas y experiencias de los actores que implementan el programa en el estado de Veracruz, México. Salud Colectiva,13(3), 521-535.

Navarrete, A. M., Barrera, M. E. y Martín, M. J. (2010). Evaluación de proyectos de servicio social en una universidad mexicana. Magis. Revista Internacional de Investigación en Educación, 2(4), 371-381. Recuperado de http://www.redalyc.org/articulo.oa?id=281021692009.

Pérez, O. A. y Pinto, R. (2020). Determinantes de la inserción laboral en egresados universitarios en México. Revista Iberoamericana para la Investigación y el Desarrollo Educativo, 11(21). Recuperado de https://doi.org/10.23913/ride.v11i21.732.

Presidencia de la República. (30 de marzo de 1981). Reglamento para la Prestación del Servicio Social de los Estudiantes de las Instituciones de Educación Superior en la República Mexicana. Diario Oficial de la Federación. Recuperado de 


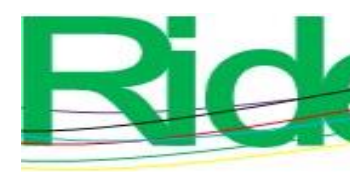

Revista Iberoamericana para la Investigación y el Desarrollo Educativo ISSN 2007 - 7467

http://www.normatecainterna.sep.gob.mx/work/models/normateca/Resource/229/1/i mages/reglamento_prestacion_servicio_social_estudiantes.pdf.

Ramírez, C. (2012). El entorno histórico en el que se dio el servicio social. Gaceta Médica de México, 148, 281-283. Recuperado de https://www.anmm.org.mx/GMM/2012/n3/GMM_148_2012_3_281-283.pdf.

Sánchez, J., Guzmán, C. G. y Baeza, G. C. (2018). Nivel de correspondencia entre la formación profesional y desempeño en el servicio social de los alumnos de la licenciatura en Enfermería de una universidad pública en Tabasco. Revista Conamed, 23(1), 17-20. Recuperado de http://www.dgdi-conamed.salud.gob.mx/ojsconamed/index.php/revconamed/article/view/599/1003.

Secretaría de Salud. (2 de septiembre de 2013). Norma Oficial Mexicana NOM-019-SSA32013. Para la práctica de enfermería en el Sistema Nacional de Salud. Diario Oficial de la Federación. Recuperado de http://www.dof.gob.mx/nota_detalle.php?codigo=5312523\&fecha=02/09/2013.

Secretaría de Salud. (3 de enero de 2017). Proyecto de Norma Oficial Mexicana PROYNOM-038-SSA3-Educación en Salud. Criterios para la utilización de los establecimientos para la atención médica como campos clínicos en la prestación del servicio social de enfermería. Diario Oficial de la Federación. Recuperado de http://www.dof.gob.mx/normasOficiales/6361/salud12_C/salud12_C.html.

Universidad Autónoma de San Luis Potosí [UASLP]. (2010). Coordinación Académica Región Altiplano. Licenciatura en Enfermería. Programa Sintético. Servicio Social. Licenciatura en Enfermería. San Luis Potosí, México: Universidad Autónoma de San Luis Potosí. Recuperado de http://www.coara.uaslp.mx/Documents/PDF_ENF/Semestre\%20IX\%20X/SERVICI O\%20SOCIAL.pdf

Universidad Autónoma de San Luis Potosí [UASLP]. (2013). Ajuste a la propuesta curricular de la Licenciatura en Enfermería de la Coordinación Académica Región Altiplano. Matehuala, México: Universidad Autónoma de San Luis Potosí. Recuperado de http://www.coara.uaslp.mx/Documents/Propuesta\%20Curricular\%20ENF.pdf.

Universidad Autónoma de San Luis Potosí [UASLP]. (2015a). Diagrama sintético del plan de estudios de la licenciatura en Enfermería. San Luis Potosí, México: Universidad 


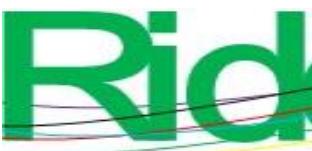

Revista Iberoamericana para la Investigación y el Desarrollo Educativo ISSN 2007 - 7467

Autónoma de San Luis Potosí. Recuperado de http://www.coara.uaslp.mx/Documents/PDF_ENF/PlanENF.pdf.

Universidad Autónoma de San Luis Potosí [UASLP]. (2015b). Reglamento del Servicio Social. San Luis Potosí, México: Universidad Autónoma de San Luis Potosí. Recuperado de http://www.uaslp.mx/Vinculacion/Documents/2017\%20Reglamento\%20del\%20Ser vicio\%20Social\%20(SO28SEP)\%20(2).pdf.

Universidad Autónoma de San Luis Potosí [UASLP]. (2017). Modelo universitario de formación integral y sus estrategias de enseñanza. San Luis Potosí, México: Universidad Autónoma de San Luis Potosí. Recuperado de http://www.uaslp.mx/Secretaria-Academica/Documents/ME/UASLPModeloEducativo2017VF.PDF. 


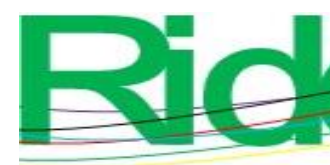

Revista Iberoamericana para la Investigación y el Desarrollo Educativo

ISSN 2007 - 7467

\begin{tabular}{|l|l|}
\hline Rol de Contribución & Autor (es) \\
\hline Conceptualización & $\begin{array}{l}\text { Alejandro Martínez Ramírez - Igual, Alma Delia Lara Cerda - } \\
\text { Principal, Ma del Rocío Rocha Rodríguez- Igual, Nereyda } \\
\text { Hernández Nava - Principal, Rosa Eréndira Fosado Quiroz - } \\
\text { Igual }\end{array}$ \\
\hline Metodología & $\begin{array}{l}\text { Alejandro Martínez Ramírez - Igual, Alma Delia Lara Cerda - } \\
\text { Igual, Ma del Rocío Rocha Rodríguez- Igual, Nereyda } \\
\text { Hernández Nava - Principal, Rosa Eréndira Fosado Quiroz - } \\
\text { Igual }\end{array}$ \\
\hline Software & NO APLICA \\
\hline Validación & $\begin{array}{l}\text { Alejandro Martínez Ramírez - Principal, Alma Delia Lara } \\
\text { Cerda - Igual, Ma del Rocío Rocha Rodríguez- Igual, Nereyda } \\
\text { Hernández Nava - Igual, Rosa Eréndira Fosado Quiroz - } \\
\text { Principal }\end{array}$ \\
\hline Análisis Formal & $\begin{array}{l}\text { Alejandro Martínez Ramírez - Principal, Alma Delia Lara } \\
\text { Cerda - Igual, Ma del Rocío Rocha Rodríguez- Igual, Nereyda } \\
\text { Hernández Nava - Igual, Rosa Eréndira Fosado Quiroz - } \\
\text { Principal }\end{array}$ \\
\hline Visualización & $\begin{array}{l}\text { Alejandro Martínez Ramírez - Igual, Alma Delia Lara Cerda - } \\
\text { Principal, Ma del Rocío Rocha Rodríguez- Igual, Nereyda } \\
\text { Hernández Nava - Principal, Rosa Eréndira Fosado Quiroz- } \\
\text { Igual }\end{array}$ \\
\hline Investigación & $\begin{array}{l}\text { Alejandro Martínez Ramírez - Principal, Alma Delia Lara } \\
\text { Cerda - Igual, Ma del Rocío Rocha Rodríguez- Igual, Nereyda } \\
\text { Hernández Nava - Igual, Rosa Eréndira Fosado Quiroz- Igual }\end{array}$ \\
\hline Escritura - Preparación del \\
edición & $\begin{array}{l}\text { Alejandro Martínez Ramírez - Principal, Alma Delia Lara } \\
\text { Cerda - Igual, Ma del Rocío Rocha Rodríguez- Igual, Nereyda } \\
\text { Hernández Nava - Igual, Rosa Eréndira Fosado Quiroz- } \\
\text { Principal }\end{array}$ \\
\hline $\begin{array}{l}\text { Alejandro Martínez Ramírez - Principal, Alma Delia Lara } \\
\text { Cerda - Igual, Ma del Rocío Rocha Rodríguez- Igual, Nereyda } \\
\text { Hernández Nava - Principal, Rosa Eréndira Fosado Quiroz- } \\
\text { Principal }\end{array}$ \\
\hline $\begin{array}{l}\text { Alejandro Martínez Ramírez - Principal, Alma Delia Lara } \\
\text { Cerda - Igual, Ma del Rocío Rocha Rodríguez- Igual, Nereyda } \\
\text { Hernández Nava - Principal, Rosa Eréndira Fosado Quiroz- } \\
\text { Principal }\end{array}$ \\
\hline
\end{tabular}




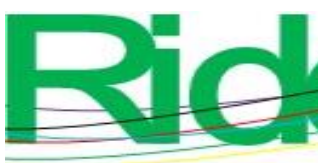

\begin{tabular}{|l|l|}
\hline & $\begin{array}{l}\text { Cerda - Igual, Ma del Rocío Rocha Rodríguez- Igual, Nereyda } \\
\text { Hernández Nava - Principal, Rosa Eréndira Fosado Quiroz- } \\
\text { Principal }\end{array}$ \\
\hline Supervisión & $\begin{array}{l}\text { Alejandro Martínez Ramírez - Igual, Alma Delia Lara Cerda - } \\
\text { Igual, Ma del Rocío Rocha Rodríguez- Igual, Nereyda } \\
\text { Hernández Nava - Principal, Rosa Eréndira Fosado Quiroz- } \\
\text { Principal }\end{array}$ \\
\hline Administración de Proyectos & $\begin{array}{l}\text { Alejandro Martínez Ramírez - Igual, Alma Delia Lara Cerda - } \\
\text { Igual, Ma del Rocío Rocha Rodríguez- Igual, Nereyda } \\
\text { Hernández Nava - Principal, Rosa Eréndira Fosado Quiroz- } \\
\text { Principal }\end{array}$ \\
\hline Adquisición de fondos & $\begin{array}{l}\text { Alejandro Martínez Ramírez - Igual, Alma Delia Lara Cerda - } \\
\text { Igual, Ma del Rocío Rocha Rodríguez- Igual, Nereyda } \\
\text { Hernández Nava - Igual, Rosa Eréndira Fosado Quiroz- Igual }\end{array}$ \\
\hline
\end{tabular}

